\title{
1 The insert sequence in SARS-CoV-2 enhances spike protein cleavage by
}

\section{TMPRSS}

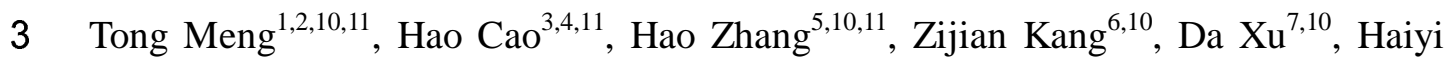

4 Gong $^{5,10}$, Jing Wang ${ }^{8}, \mathrm{Zifu}^{2}{ }^{8}$, Xingang Cui ${ }^{7}$, Huji Xu ${ }^{4,6}$, Haifeng Wei ${ }^{5}$, Xiuwu Pan ${ }^{7}$,

5 Rongrong Zhu ${ }^{9}$, Jianru Xiao ${ }^{5 *}$, Wang Zhou $^{4,10} *$, Liming Cheng ${ }^{1} *$, Jianmin Liu $^{8 *}$.

61 Division of Spine, Department of Orthopedics, Tongji Hospital affiliated to Tongji

7 University School of Medicine, 200065 Shanghai, China

82 Tongji University Cancer Center, School of Medicine, Tongji University, 200092

9 Shanghai, China

103 School of Life Science and Biopharmaceutics, Shenyang Pharmaceutical University,

11103 Wenhua Road, 110016 Shenyang, China

124 Peking-Tsinghua Center for Life Sciences, TsinghuaUniversity, 100084 Beijing,

13 China

145 Department of Orthopaedic Oncology, Changzheng Hospital, Second Military

15 Medical University, 200003 Shanghai, China

166 Department of Rheumatology and Immunology, Changzheng Hospital, Second

17 Military Medical University, 200003 Shanghai, China

187 Depanrtment of Urology, The Third Affiliated Hospital of Second Military Medical

19 University, 201805 Shanghai, China

208 Department of Neurosurgery, Changhai hospital, Second Military Medical

21 University, 200003 Shanghai, China 
19 Key Laboratory of Spine and Spinal Cord Injury Repair and Regeneration of

2 Ministry of Education, Orthopaedic Department of Tongii Hospital, School of Life

3 Science and Technology, Tongji University, 200092 Shanghai, China

410 Qiu-Jiang Bioinformatics Institute, 200003 Shanghai, China

511 These authors contributed equally to this work: Tong Meng, Hao Cao, Hao Zhang

6 *e-mail: chstroke@163.com; limingcheng@tongji.edu.cn; brilliant212@163.com;

7 jianruxiao83@163.com

8 Abstract

9 At the end of 2019, the SARS-CoV-2 induces an ongoing outbreak of pneumonia in

10 China $^{1}$, even more spread than SARS-CoV infection ${ }^{2}$. The entry of SARS-CoV into

11 host cells mainly depends on the cell receptor (ACE2) recognition and spike protein

12 cleavage-induced cell membrane fusion ${ }^{3,4}$. The spike protein of SARS-CoV-2 also

13 binds to ACE2 with a similar affinity, whereas its spike protein cleavage remains

14 unclear $^{5,6}$. Here we show that an insertion sequence in the spike protein of

15 SARS-CoV-2 enhances the cleavage efficiency, and besides pulmonary alveoli,

16 intestinal and esophagus epithelium were also the target tissues of SARS-CoV-2.

17 Compared with SARS-CoV, we found a SPRR insertion in the S1/S2 protease

18 cleavage sites of SARS-CoV-2 spike protein increasing the cleavage efficiency by the

19 protein sequence aligment and furin score calculation. Additionally, the insertion

20 sequence facilitates the formation of an extended loop which was more suitable for

21 protease recognition by the homology modeling and molicular docking. Furthermore, 
1 the single-cell transcriptomes identified that ACE2 and TMPRSSs are highly

2 coexpressed in AT2 cells of lung, along with esophageal upper epithelial cells and

3 absorptive enterocytes. Our results provide the bioinformatics evidence for the

4 increased spike protein cleavage of SARS-CoV-2 and indicate its potential target

5 cells.

\section{Introduction}

7 At the end of 2019, a rising number of pneumonia patients with unknown pathogen

8 emerged from Wuhan to nearly the entire China ${ }^{7}$. A novel coronavirus was isolated

9 and based on its phylogeny, taxonomy and established practice, the Coronavirus

10 Study Group (CSG) recognized it as a sister to severe acute respiratory syndrome

11 coronaviruses (SARS-CoVs) and labeled it as severe acute respiratory syndrome

12 coronavirus $2(\mathrm{SARS}-\mathrm{CoV}-2)^{1,8}$. Although SARS-CoV-2 is generally less pathogenic

13 than SARS-CoV and Middle East respiratory syndrome coronavirus (MERS-CoV), it

14 has a relatively high transmissibility ${ }^{9}$.

15 With regard to human coronavirus, the transmissibility and infectivity is largely

16 controlled by the spike (S) surface envelope protein ${ }^{10}$. Its surface unit (S1) mediates

17 the entry into host cells by binding to cell receptor and the transmembrane unit (S2)

18 subunit regulates the fusion of viral and cellular membranes ${ }^{3}$. Prior to membrane

19 fusion, the $\mathrm{S}$ protein should be cleaved and activated to allow for the fusion peptide

20 releasing onto host cell membranes (Fig. 1a) ${ }^{11}$. SARS-CoV-2 uses the same cell

21 receptor (angiotensin converting enzyme II, ACE2) as SARS-CoV, with a similar 
1 binding affinity, whereas their transmissibility and infectivity are different ${ }^{5,6,12,13}$.

2 Thus, the different virus transmission and infectivity may be associated with the

3 differentiated protease-induced S protein cleavage between SARS-CoV-2 and

4 SARS-CoV.

5 The transmembrane serine proteases (TMPRSSs) were the main host cell proteases on

6 the cell membrane ${ }^{14}$. The substrate specificity of TMPRSSs are almost similar and

7 revealing a strong preference for arginine or lysine residues in the P1 position.

8 Nowadays, their hydrolytic effects of TMPRSSs have been widely reported in

9 SARS-CoV and MERS-CoV pneumonia ${ }^{15}$. In the SARS-CoV-infected alveolar cells,

10 TMPRSSs, especially the TMPRSS2 and TMPRSS11D, cleave the SARS-CoV S

11 protein (SARS-S) at residue R667 (the S1/S2 cleavage site) and residue R797 (the S2'

12 cleavage site) (Fig. 1a) ${ }^{15,16}$. Besides cleaving S protein, they can also promote viral

13 spread in the host by cleaving ACE2 (Fig. 1b) ${ }^{14,17}$. Although SARS-CoV-2 and

14 SARS-CoV share the same host cell receptor with a similar affinity, however, the

15 SARS-CoV-2 S protein cleavage induced by TMPRSS remains unclear which may be

16 associated with the viral infectivity ${ }^{4,5}$.

\section{Results}

18 The comparison of the S1/S2 and S2' cleavage sites between SARS-CoV-2 and

19 SARS-CoV

20 Generally, compared with SARS-CoV, the major differences in SARS-CoV-2 are the

21 three short insertions in the N-terminal domain and four out of five key residues 
1 changes in the receptor-binding motif ${ }^{5}$. Here we used the alignment, furin score and

2 homology modeling to compare the sequence of the S1/S2 and S2' cleavage sites

3 between SARS-CoV-2 and SARS-CoV (Fig. 1c). The amino acid sequence of the

4 S1/S2 and S2' cleavage sites among ten beta-coronavirus were then analyzed and we

5 found that compared with SARS, there was an insertion sequence (SPRR) in the

6 S1/S2 cleavage sites of SARS-CoV-2 (Fig. 2a). The furin score was next used to

7 identify the cleavage efficiency of the insertion sequence in SARS-CoV-2. Its furin

8 score was 0.688 , which was obviously higher than that of the corresponding sequence

9 in SARS-CoV (0.139), indicating that the insertion sequence may increase the

10 cleavage efficiency by proteases (Fig. 2b).

11 The structures of SARS-S and SARS-CoV-2 S protein were presented in Extended

12 Data Fig. 1a and 1b, along with their structural superimposition (Extended Data Fig.

13 1c). The structural comparison of homology modeling SARS-CoV-2 S protein with

14 SARS-S protein (PDB: 5x5b) showed that a exposed loop was formed by the insertion

15 which comprised R682 and R683 (S1/S2 site) on the surface of SARS-CoV-2 S

16 protein, and no significant difference of them in S2' site (Fig. 2c, d).

17 The insertion sequence of SARS-CoV-2 facilitating the TMPRSS recognition and

18 S protein cleavage

19 Structurally, TMPRSSs include extracellular domain, transmembrane domain and

20 intracellular domain in which extracellular domain is the main catalytic domain. They

21 show similar substrate-specificity and catalytic mechanism. Take TMPRSS2 as an 
1 example. The catalytic triad consisted of H296, D345 and S441 and the substrate

2 binding residue D435, a conserved aspartate residue, was located in the bottom of

3 pocket $^{18,19}$. The substrate binding pocket is deeper than most of serine proteinase

4 (Extended Data Fig. 2a, b). The bottom of the catalytic pocket has a negatively

5 charged aspartic acid residue which can facilitate the binding and stabilization of

6 arginine or lysine residues in the $\mathrm{P} 1 \operatorname{position}^{18,19}$.

7 Polypeptide substrate analogue KQLR included arginine, glutamine, leucine and

8 lysine (Extended Data Fig. 2c). The substrate analogue could bind to the catalytic

9 pocket of TMPRSS2 (Extended Data Fig. 2d, e). The conformation of the insertion

10 sequence in SARS-CoV-2 S protein and TMPRSS2 was next simulated by molecular

11 docking. We found the insertion sequence formed a loop which was easily recognized

12 by the catalytic pocket of TMPRSS2 (Extended Data Fig. 2f, g). Thus, both the furin

13 score and molecular docking revealed that the insertion sequence of SARS-CoV-2

14 facilitates the TMPRSS2 recognition and S protein cleavage.

15 The potential target tissues of COVID-19

16 The entry of SARS-CoV-2 into host cells depends on the cell receptor recognition and

17 cell proteases cleaving. Thus, the target cells should coexpress both the cell receptor

18 ACE2 and cell proteases TMPRSSs. In order to identify the coexpressing cell

19 composition and proportion, we utilized 3 datasets including 32 samples and built the

20 largest single-cell transcriptome atlas of normal lung, the commonest infected organ

21 of SARS-CoV-2. 
1 After initial quality controls, a total of 113,045 cells and 29 sub-clusters were

2 identified in the lung (Fig. 3a). The marker genes and dataset proportions of each

3 sub-cluster were presented in Extended Data Fig. 3-4.

4 We detected the expression of ACE2 and TMPRSSs in 29 cell groups, in which the

5 expression of the whole 17 TMPRSS genes is in the form of total signature value.

6 Pseudodyeing analysis was performed and we found that ACE2 was mainly expressed

7 in AT2 cells and marked with red (Fig. 3b, c). The total 17 TMPRSS genes was found

8 in AT1, AT2, airway secretory and ciliated cells colored with blue (Fig. 3b, d,

9 Extended Data Fig. 5a). Thus, we found an obvious coexpression between TMPRSSs

10 and ACE2 in AT2. Among the whole TMPRSS genes, TMPRSS1 and TMPRSS2

11 were highly expressed in AT2 and AT1 cells, which were co-expressed with ACE2 in

12 lung (Fig. 3b, Extended Data Fig. 5b). Due to the entry of virus into host cell is

13 related to endocytosis, we also detected the endocytosis-related genes among different

14 cells. We found that these genes had consistent distribution and highly expressed in

15 AT1, AT2, airway secretory, ciliated cells and M2 macrophage (Extended Data Fig.

$165 c)$

17 Due to the RNA of SARS-CoV-2 was also found in the stool specimen of the

18 SARS-CoV-2-infected patient ${ }^{20}$, the digestive system may also be the potential route

19 of COVID-19. Thus, in addition to lung, 4 datasets with the single-cell transcriptomes

20 of the esophagus, gastric, small intestine and colon were analyzed to identify the

21 expression of ACE2 and TMPRSSs in the digestive system. The co-expression of 
1 ACE2 and TMPRSS was analyzed in esophagus, stomach, small intestine and colon

2 by $87947,29678,11218$ and 47442 high-quality single cells, respectively (Extended

3 Data Fig. 6a). The coexpression of ACE2 and total TMPRSS genes were found in the

4 upper epithelial cells of esophagus, the absorptive enterocytes of ileum epithelia and

5 the enterocytes of colon epithelia (Extended Data Fig. 6b-e, 7a-d).

6 As both ACE2 and TMPRSSs are expressed in the lung and digestive system, we next

7 compared their relative expression values in the ACE2-expressing cells. A similar

8 distribution was found between ACE2 and TMPRSSs in all the 9 clusters with high

9 expressions in the esophageal upper epithelial cells, the ileal absorptive enterocytes

10 and the colonic enterocytes (Fig. 4a). In addition, their expression of AT2 was

11 relatively lower than that of epithelial cells in the digestive system. Among all the

12 TMPRSSs, TMPRSS1 and TMPRSS2 were relatively highly expressed in AT2, and

13 most TMPRSSs were highly found in the esophageal upper epithelial cells (Extended

14 Data Fig. 8a). The endocytosis- and exocytosis-associated genes which are related to

15 the entry of virus into host cells and virus infection were also detected in all the 9

16 clusters. The endocytosis signature was more expressed in AT1 and AT2 cells,

17 whereas the exocytosis signature was highly gathered in esophageal upper epithelial

18 cells. It can explain that the commonest infected tissue in COVID-19 is pulmonary

19 alveoli and SARS-CoV-2 can also be detected in the esophageal erosion (Fig. 4b) ${ }^{21}$.

20 The RNA-seq data of lung, esophagus, stomach, small intestine, colon-transverse and

21 colon-sigmoid were obtained from GTEx database. The expressions of ACE2 and 
1 TMPRSS2 also had a similar tendency and were highly expressed in small intestine

2 and colon, while the TMPRSS11D was mainly found in the esophagus (Extended

3 Data Fig. 8b).

4 Discussion

5 The coronaviruses is the common infection source of respiratory, enteric and central

6 nervous system in humans and other mammals ${ }^{22}$. At the beginning of the twenty-first

7 century, two betacoronaviruses, SARS-CoV and MERS-CoV, result in persistent

8 public panics and became the most significant public health events ${ }^{23}$. In December

9 2019, a novel identified coronavirus (SARS-CoV-2) induced an ongoing outbreak of

10 pneumonia in Wuhan, Hubei, China ${ }^{7}$. The rapidly increasing number of

11 SARS-CoV-2-infected cases suggests that SARS-CoV-2 may be transmitted

12 effectively among humans and give rise to a high pandemic potential ${ }^{7,8,24}$.

13 Previous studies identified that SARS-CoV mutated between 2002 and 2004 to better

14 bind to its cell receptor, replicate in human cells and enhance the virulence ${ }^{9}$. Thus, it

15 is important to explore whether SARS-CoV-2 behaves like SARS-CoV to adapt to the

16 host cell. Notably, SARS-CoV and SARS-CoV-2 share the same receptor protein

$17 \mathrm{ACE} 2^{5,25}$. Besides, the receptor-binding domain (RBD) in S protein of SARS-CoV-2

18 binds to ACE2 with the similar affinity as SARS-CoV RBD does ${ }^{6}$. Thus, during the

19 process of viral and host cellular membrane fusion, whether the specific structure of

20 SARS-CoV-2 S protein seems better suited to be activated by host cell proteases may 
1 be related to the different virus infectivities and transmissibilities between

\section{SARS-CoV and SARS-CoV-2 $2^{6}$.}

3 In this study, we found the furin score of the S1/S2 cleavage sites in SARS-CoV-2

4 was higher than that of SARS, implying a more degree of hydrolysis. Through the

5 comparison of the two structures, R682, R683 and relative S680, P681 extended the

6 original exposed loop combined with R685 of SARS-CoV-2, which was more

7 suitable for hydrolysis by TMPRSSs. The substrate specificity of TMPRSSs are

8 almost similar, revealing a strong preference for arginine or lysine residues in the P1

9 position represented by R. More R (R682, R683 and R685) in the S1/S2 cleavage

10 sites of SARS-CoV-2 can enhance the cleavage of S1 with S2, which means that the

11 structurally constrains of S1 on S2 is removed, and the fusion peptides in S2 are

12 exposed and insert into the target host cell membrane, finally it increases the

13 efficiency of fuse membranes ${ }^{18,19}$.

14 By the way, some researchers previously supposed the SARS-CoV-2 was artificial

15 due to four inserts in the S protein of SARS-CoV-2 from HIV sequence. However, the

16 results of protein sequence alignment revealed that the similar sequence of the

17 reported fourth insertion site (680-SPRR-683) in SARS-CoV-2 was commonly found

18 in many beta-coronavirus. Therefore, we supposed that based on the current evidence,

19 it is not scientific to consider the insertion sequence in SARS-CoV-2 S protein being

20 artificial. 
1 With the help of single cell sequencing, we found a strong co-expression between

2 ACE2 and TMPRSSs, in especial TMPRSS1and TMPRSS2, in lung AT2 cells, which

3 was also the main infected cell type in SARS-CoV pneumonia ${ }^{26}$. Moreover, we also

4 found the endocytosis-associated genes was highly expressed in AT2 cells, implying

5 that endocytosis may also facilitate the entry of SARS-CoV-2 into host cells. As the

6 alveolar stem-like cells, AT2 cells are in charge of surfactant biosynthesis,

7 self-renewal and immunoregulation ${ }^{27}$. Thus, SARS-CoV-2 not only damages the AT2

8 cells leading to the direct injury to alveoli, but also raises alveolar surface tension to

9 induce dyspnea ${ }^{28}$. Additionally, the injuryed AT2 also damages the immunologic

10 balance in alveoli and results in inflammatory cascade ${ }^{29}$. In addition, they are also

11 highly co-expressed in absorptive enterocytes and upper epithelial cells of esophagus,

12 implying that intestinal epithelium and esophagus epithelium may also be the

13 potential target tissues. This can explain the cases whose SARS-CoV-2 was detected

14 in the esophageal erosion or stool specimen, implying that the digestive system is a

15 potential route of COVID-19 ${ }^{7,20,21}$.

16 Due to the critical role of TMPRSSs in influenza virus and coronavirus infections,

17 serine protease inhibitors, such as camostat, nafamostat and leupeptin, have been used

18 in the antiviral treatment targeting TMPRSSs with high antiviral activities ${ }^{14,30,31}$.

19 Nowadays, Remdesivir (GS-5734) has been used in the treatment of SARS-CoV-2,

20 however, the therapeutic effects are still unclear. Based on our results, we also

21 supposed that TMPRSSs may also serve as candidate antiviral targets for 
1 SARS-CoV-2 infection and the clinical trials of serine protease inhibitors should also

2 be performed for COVID-19.

\section{Methods}

\section{$4 \quad$ Structure modelling}

5 The structures of SARS-CoV-2 S protein and TMPRSS2 were generated by

6 SWISS-MODEL online server ${ }^{32}$. The structures were marked, superimposed and

$7 \quad$ visualized by Chimera ${ }^{33}$. To further explore the possible catalytic mechanism of the

8 SARS-CoV-2 S protein cleaved by TMPRSS2, ZDOCK program was used to predict

9 their interaction ${ }^{34}$. A total of 5000 models were generated and were set to 50 clusters,

10 then the best scoring models from the 5 largest clusters were selected for further

11 analysis.

\section{Furin score}

13 The fragmentation maps, scoring and residue coverage analysis were conducted using

14 arginine and lysine propeptide cleavage sites prediction algorithms ProP 1.0 server $^{35}$.

\section{Single cell transcriptome data sources}

16 Single cell transcriptome data were obtained from Single Cell Portal

17 (https://singlecell.broadinstitute.org/single_cell), Human Cell Atlas Data Protal

18 (https://data.humancellatlas.org) and Gene Expression Omnibus (GEO;

19 https://www.ncbi.nlm.nih.gov/). Esophageal and lung data were obtained from the

20 research of E Madissoon et al containing 21 esophageal and 19 lung tissue samples ${ }^{36}$.

21 Two lung datasets were further obtained from GSE122960 38 and GSE128169 $9^{39}$, 
1 including eight and five lung tissues respectively. GSE134520 included 6 gastric

2 mucosal samples from 3 non-atrophic gastritis and 2 chronic atrophic gastritis

3 patients $^{40}$. GSE134809 comprises 11 noninflammatory ileal samples from Crohn's

4 disease patients ${ }^{41}$. The data from Christopher $\mathrm{S}$ et al consisted of 12 normal colon

5 samples $^{42}$.

\section{Quality control}

7 Cells would be identified as poor-quality once (1) the number of expressed genes

8 fewer than 200 or greater than 5000, or (2) more than $20 \%$ of UMIs being mapped to

9 mitochondrial or ribosomal genes.

\section{Data Integration, Dimension Reduction and Cell Clustering}

11 Different methods were performed to process the downloaded data:

12 1. Esophagus dataset. Rdata were obtained and dimension reduction and clustering

13 had already been implemented by the authors ${ }^{36}$.

14 2. Lung, stomach and ileum datasets. We utilized functions in the Seurat package to

15 normalize and scale the single-cell gene expression data ${ }^{43}$. Unique

16 molecularidentifier (UMI) counts were normalized by the total number of UMIs

17 per cell, multiplied by 10000 for normalization and log-transformed using the

18 NormalizeData" function. Then, multiple sample data within each dataset were

19 merged using the "FindIntegrationAnchors" and "Integratedata" functions. After

20 identifying highly variable genes (HVGs) using the "FindVariableGenes" function

21 a principal component analysis (PCA) was performed on the single-cell 

21 impute lost values in the gene expression matrix. The imputed gene expression was

expression matrix using the "RunPCA" function. The "FindClusters" function in the Seurat package was next utilized to conduct the cell clustering analysis into a graph structure in PCA space after constructing a K-nearest-neighbor graph based on the Euclidean distance in PCA space. Uniform Manifold Approximation and Projection (UMAP) visualization was performed for obtaining the clusters of cells.

3. Colon Dataset. The single cell data was processed with the R packages LIGER ${ }^{44}$ and Seurat ${ }^{43}$. The gene expression matrix was first normalized to remove differences in sequencing depth and capture efficiency among cells. Variable genes in each dataset were identified using the "selectGenes" function. Then we used the "optimizeALS" function in LIGER to perform the integrative nonnegative matrix factorization and selecte a $\mathrm{k}$ of 15 and lambda of 5.0 to obtain a plot of expected alignment. The "quantileAlignSNF" function was then performed to builds a shared factor neighborhood graph to jointly cluster cells, then quantile normalizes corresponding clusters. Next nonlinear dimensionality reduction was calculated using the "RunUMAP" function and the results were visualized with UMAP.

\section{Identification of cell types and Gene expression analysis}

Clusters were annotated on the expression of known cell markers and the clustering information provided in the articles. Then, we utilized the "RunALRA" function to 
1 shown in Feature plots and violin plots. We used "Quantile normalization" in the R

2 package preprocessCore ( $\mathrm{R}$ package version 1.46.0.

$3 \mathrm{https}: / /$ github.com/bmbolstad/preprocessCore) to remove unwanted technical

4 variability across different datasets. The data were further denoised to compare the

5 gene expression levels of gene signature.

6 Endocytosis or exocytosis associated genes were obtained from Harmonizome dataset

$7 \quad{ }^{45}$.Mean expressions of the genesets were calculated to compare the ability of

8 endocytosis or exocytosis among clusters.

9 To minimize bias, external databases of Genotype-Tissue Expression (GTEx) ${ }^{46}$ was

10 used to detect gene expression of ACE2, TMPRSS1 and TMPRSS2 at the tissue

11 levels including normal lung and digestive system, such as esophagus, stomach, small

12 intestine and colon.

14 Acknowledgements This study was jointly supported by the National Natural

15 Science Foundation of China (Grants 81702659 and 81572746) and National Key

16 R\&D Program of China (Grants 2016YFA0100800).

17 Author contributions J.L., L.C., W.Z. and J.X. conceived the idea and directed the

18 team. T.M., H.C., H.Z. and W.Z. designed and coordinated the analysis and

19 characterization. H.Z., Z.K., D.X., H.G. performed single-cell sequencing and

20 characterization under the guidance of X.C., H.X., and H.W.. Data collection and

21 generation were performed by J.W., Z.L., R.Z. and X.P.. Data interpretation was 
1 performed by J.L., L.C., W.Z. and J.X.. The alignment and structure comparison was

2 performed by H.C. under the guidance of W.Z. The manuscript was written by T.M.,

3 H.C., Z.K. and W.Z. All authors contributed to the analysis and discussion of the

4 results leading to the manuscript.

5 Competing interests The authors declare no competing interests.

6

7

81 Gorbalenya AE, B. S., Baric RS, de Groot RJ, Drosten C, Gulyaeva AA,

9 Haagmans BL, Lauber C, Leontovich AM, Neuman BW, Penzar D,

Perlman S, Poon LL, Samborskiy D, Sidorov IA, Sola I, Ziebuhr J. Severe acute respiratory syndrome-related coronavirus: The species and its viruses - a statement of the Coronavirus Study Group. bioRxiv doi:doi: https://doi.org/10.1101/2020.02.07.937862 (2020).

142 Zhong, N. S. et al. Epidemiology and cause of severe acute respiratory syndrome (SARS) in Guangdong, People's Republic of China, in February, $\quad 2003$. Lancet 362 1353-1358, doi:10.1016/s0140-6736(03)14630-2 (2003).

183 Hofmann, H. \& Pohlmann, S. Cellular entry of the SARS coronavirus. Trends in microbiology 12, 466-472, doi:10.1016/j.tim.2004.08.008 (2004).

214 Chan, J. F. et al. A familial cluster of pneumonia associated with the 
178 Zhu, N. et al. A Novel Coronavirus from Patients with Pneumonia in 18 China, 2019. The New England journal of medicine,

209 Chen, J. Pathogenicity and Transmissibility of 2019-nCoV-A Quick

2019 novel coronavirus indicating person-to-person transmission: a study of a family cluster. Lancet, doi:10.1016/s0140-6736(20)30154-9 (2020).

P Zhou, X. Y., XG Wang, B Hu, L Zhang, W Zhang, HR Si, Y Zhu, B Li, CL Huang, HD Chen, J Chen, Y Luo, H Guo, RD Jiang, MQ Liu, Y Chen, XR Shen, X Wang, XS Zheng, K Zhao, QJ Chen, F Deng, LL Liu, B Yan, FX Zhan, YY Wang, GF Xiao, ZL Shi. A pneumonia outbreak associated with a new coronavirus of probable bat origin. Nature, doi:https:// doi.org/10.1038/s41586-020-2012-7 (2020).

Tian XL, L. C., Huang A, Xia S, Lu SC, Shi ZL, Lu L, Jiang SB, Yang ZL, Wu YL, Ying TL. Potent binding of 2019 novel coronavirus spike protein by a SARS coronavirus-specific human monoclonal antibody. bioRxiv doi:doi: https://doi.org/10.1101/2020.01.28.923011 (2020).

Huang, C. et al. Clinical features of patients infected with 2019 novel coronavirus in Wuhan, China. Lancet, doi:10.1016/s0140-6736(20)30183-5 (2020). doi:10.1056/NEJMoa2001017 (2020). Overview and Comparison with Other Emerging Viruses. Microbes and 
infection, doi:10.1016/j.micinf.2020.01.004 (2020).

210 Walls, A. C. et al. Unexpected Receptor Functional Mimicry Elucidates Activation of Coronavirus Fusion. Cell 176, 1026-1039.e1015, doi:10.1016/j.cell.2018.12.028 (2019).

511 Gallagher, T. M. \& Buchmeier, M. J. Coronavirus spike proteins in viral entry and pathogenesis. Virology 279, 371-374, doi:10.1006/viro.2000.0757 (2001).

812 Gui, M. et al. Cryo-electron microscopy structures of the SARS-CoV spike glycoprotein reveal a prerequisite conformational state for receptor binding. Cell research 27, 119-129, doi:10.1038/cr.2016.152 (2017). Preliminary estimation of the basic reproduction number of novel coronavirus (2019-nCoV) in China, from 2019 to 2020: A data-driven analysis in the early phase of the outbreak. . bioRxiv, 916395, doi:doi: https://doi.org/10.1101/2020.01.23.916395 (2020).

1714 Zhou, Y. et al. Protease inhibitors targeting coronavirus and filovirus entry. Antiviral research 116, 76-84, doi:10.1016/j.antiviral.2015.01.011 (2015).

2015 Millet, J. K. \& Whittaker, G. R. Host cell proteases: Critical determinants of coronavirus tropism and pathogenesis. Virus research 202, 120-134, 
doi:10.1016/j. virusres.2014.11.021 (2015).

216 Shirato, K., Kawase, M. \& Matsuyama, S. Wild-type human coronaviruses prefer cell-surface TMPRSS2 to endosomal cathepsins for cell entry. Virology 517, 9-15, doi:10.1016/j.virol.2017.11.012 (2018).

617 Heurich, A. et al. TMPRSS2 and ADAM17 cleave ACE2 differentially and only proteolysis by TMPRSS2 augments entry driven by the severe acute respiratory syndrome coronavirus spike protein. Journal of virology 88, 1293-1307, doi:10.1128/jvi.02202-13 (2014). for human hepsin, a membrane-anchored serine protease implicated in prostate and ovarian cancers. The Biochemical journal 390, 125-136, doi:10.1042/bj20041955 (2005).

1419 Limburg, $H$. et al. TMPRSS2 is the Major Activating Protease of Influenza A Virus in Primary Human Airway Cells and Influenza B Virus in Human Type II Pneumocytes. Journal of virology 93, doi:10.1128/jvi.00649-19 (2019).

1820 Holshue, M. L. et al. First Case of 2019 Novel Coronavirus in the United States. The New England journal of medicine, doi:10.1056/NEJMoa2001191 (2020).

2121 Guan WJ, N. Z., Hu Y, Liang WH, Ou CQ, He JX, Liu L, Shan H, Lei CL, 
1 Hui David S.C., Du B Clinical characteristics of 2019 novel

2 coronavirus infection in China. medRxiv, doi:doi:

$3 \quad$ http://dx.doi.org/10.1101/2020.02.06.20020974 (2020).

422 Perlman, S. \& Netland, J. Coronaviruses post-SARS: update on

5 replication and pathogenesis. Nature reviews. Microbiology 7, 439-450,

6 doi:10.1038/nrmicro2147 (2009).

723 de Wit, E., van Doremalen, N., Falzarano, D. \& Munster, V. J. SARS

8 and MERS: recent insights into emerging coronaviruses. Nature

9 reviews. Microbiology 14, 523-534, doi:10.1038/nrmicro.2016.81

$10 \quad(2016)$

1124 Lee, P. I. \& Hsueh, P. R. Emerging threats from zoonotic

12 coronaviruses-from SARS and MERS to 2019-nCoV. Journal of

13 microbiology, immunology, and infection = Wei mian yu gan ran za zhi,

14 doi:10.1016/j.jmii.2020.02.001 (2020).

$1525 \mathrm{Li}, \mathrm{W}$. et al. Angiotensin-converting enzyme 2 is a functional receptor for

16 the SARS coronavirus. Nature 426, 450-454, doi:10.1038/nature02145

17 (2003).

1826 Kuiken, T. et al. Newly discovered coronavirus as the primary cause of

19 severe acute respiratory syndrome. Lancet 362, 263-270,

20 doi:10.1016/s0140-6736(03)13967-0 (2003).

2127 Nabhan, A. N., Brownfield, D. G., Harbury, P. B., Krasnow, M. A. \& 
Desai, T. J. Single-cell Wnt signaling niches maintain stemness of alveolar type 2 cells. Science (New York, N.Y.) 359, 1118-1123, doi:10.1126/science.aam6603 (2018).

428 Barkauskas, C. E. et al. Type 2 alveolar cells are stem cells in adult lung.

5 The Journal of clinical investigation 123, 3025-3036,

6 doi:10.1172/jci68782 (2013).

729 Kroetz, D. N. et al. Type I Interferon Induced Epigenetic Regulation of

8 Macrophages Suppresses Innate and Adaptive Immunity in Acute

9 Respiratory Viral Infection. PLOS pathogens 11, e1005338, 10 doi:10.1371/journal.ppat.1005338 (2015).

1130 Shen, L. W., Mao, H. J., Wu, Y. L., Tanaka, Y. \& Zhang, W. TMPRSS2:

12 A potential target for treatment of influenza virus and coronavirus 13 infections. Biochimie 142, 1-10, doi:10.1016/j.biochi.2017.07.016 14 (2017).

1531 Shin, W. J. \& Seong, B. L. Type II transmembrane serine proteases as potential target for anti-influenza drug discovery. Expert opinion on drug 17 discovery 12, 1139-1152, doi:10.1080/17460441.2017.1372417 (2017).

1832 Biasini, M. et al. SWISS-MODEL: modelling protein tertiary and quaternary structure using evolutionary information. Nucleic acids research 42, W252-258, doi:10.1093/nar/gku340 (2014).

2133 Pettersen, E. F. et al. UCSF Chimera--a visualization system for 
exploratory research and analysis. Journal of computational chemistry 25, 1605-1612, doi:10.1002/jcc.20084 (2004).

334 Wiehe, K. et al. ZDOCK and RDOCK performance in CAPRI rounds 3, 4, and 5. Proteins 60, 207-213, doi:10.1002/prot.20559 (2005).

535 Duckert, P., Brunak, S. \& Blom, N. Prediction of proprotein convertase cleavage sites. Protein engineering, design \& selection : PEDS 17, 107-112, doi:10.1093/protein/gzh013 (2004).

836 Madissoon, E. et al. scRNA-seq assessment of the human lung, spleen, and esophagus tissue stability after cold preservation. Genome biology 21, 1, doi:10.1186/s13059-019-1906-x (2019).

1137 Vieira Braga, F. A. et al. A cellular census of human lungs identifies novel cell states in health and in asthma. Nature medicine 25, 1153-1163, doi:10.1038/s41591-019-0468-5 (2019).

1438 Reyfman, P. A. et al. Single-Cell Transcriptomic Analysis of Human Lung Provides Insights into the Pathobiology of Pulmonary Fibrosis. American joumal of respiratory and critical care medicine 199, 1517-1536, doi:10.1164/rccm.201712-24100C (2019).

1839 Valenzi, E. et al. Single-cell analysis reveals fibroblast heterogeneity and myofibroblasts in systemic sclerosis-associated interstitial lung disease. Annals of the rheumatic diseases 78, 1379-1387, doi:10.1136/annrheumdis-2018-214865 (2019). 
140 Zhang, P. et al. Dissecting the Single-Cell Transcriptome Network

$2 \quad$ Underlying Gastric Premalignant Lesions and Early Gastric Cancer.

3 Cell reports 27, 1934-1947.e1935, doi:10.1016/j.celrep.2019.04.052

$4 \quad$ (2019).

541 Martin, J. C. et al. Single-Cell Analysis of Crohn's Disease Lesions

6 Identifies a Pathogenic Cellular Module Associated with Resistance to<smiles>[3H][V]</smiles>
Anti-TNF Therapy. Cell 178, 1493-1508.e1420, doi:10.1016/j.cell.2019.08.008 (2019).

942 Smillie, C. S. et al. Intra- and Inter-cellular Rewiring of the Human Colon during Ulcerative Colitis. Cell 178, 714-730.e722, doi:10.1016/j.cell.2019.06.029 (2019).

1243 Stuart, T. et al. Comprehensive Integration of Single-Cell Data. Cell 177, 13 1888-1902.e1821, doi:10.1016/j.cell.2019.05.031 (2019).

1444 Welch, J. D. et al. Single-Cell Multi-omic Integration Compares and 15 Contrasts Features of Brain Cell Identity. Cell 177, 1873-1887.e1817, 16 doi:10.1016/j.cell.2019.05.006 (2019).

1745 Rouillard, A. D. et al. The harmonizome: a collection of processed 18 datasets gathered to serve and mine knowledge about genes and 19 proteins. Database : the joumal of biological databases and curation 20 2016, doi:10.1093/database/baw100 (2016).

2146 Human genomics. The Genotype-Tissue Expression (GTEx) pilot 
347 Uhlen, M. et al. Proteomics. Tissue-based map of the human proteome.

$\mathbf{a}$
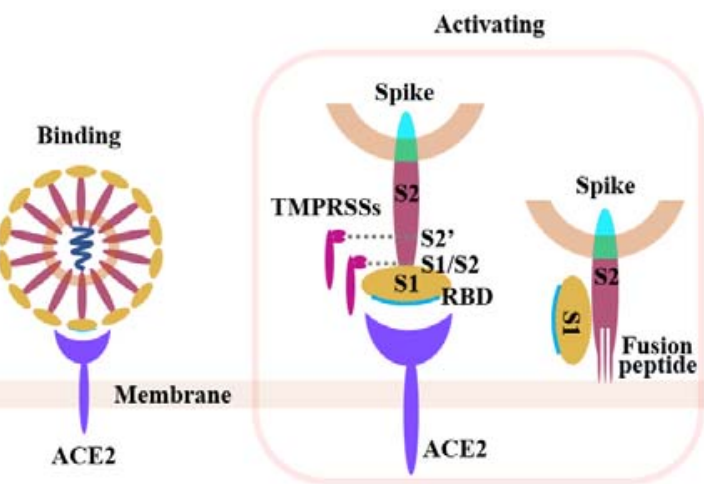

Entry and uncoating

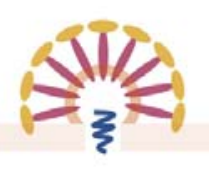

b

Binding



c
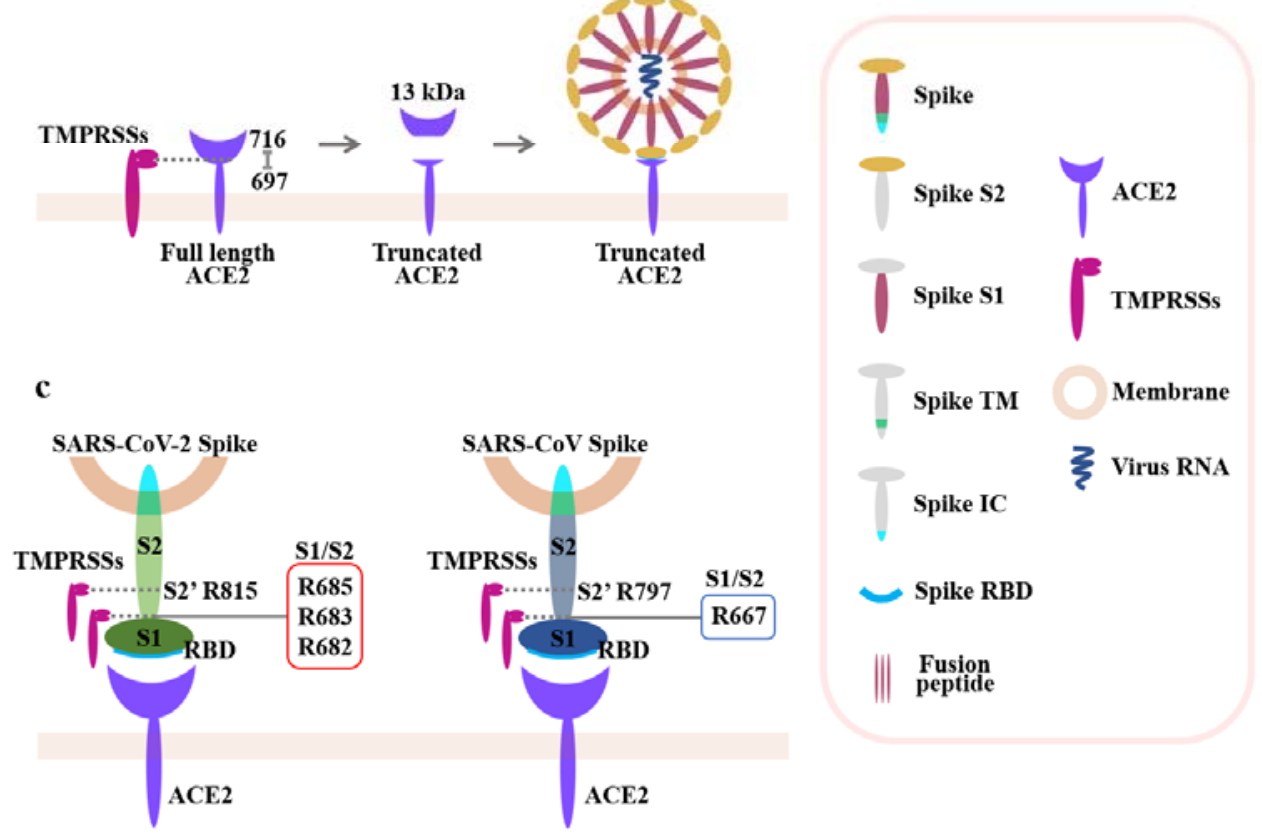

7

8 Fig. 1 The schematic diagram of the project.

9 a. The entry of SARS-CoV into host cells: The spike protein of SARS-CoV binds to 
1 ACE2 through its S1 subunit for viral recognition. Then it is cleaved by

2 TMPRSS2 at the S1/S2 boundary or within S2 subunit, which removes the

3 structural constraint of $\mathrm{S} 1$ on $\mathrm{S} 2$, and releases the internal fusion peptide

4 combined with the Spike TM domain for the fusion of viral and cellular

5 membranes. Finally, the viral genomes enter into the host cells.

6 b. ACE2 cleaving by TMPRSSs: TMPRSS2 can also cleave ACE2 amino acids 697

7 to 716 , resulting in the shedding of $13 \mathrm{kD}$ ACE2 fragment in culture supernatants

8 and augmented viral infectivity.

9 c. The difference between SARS-CoV-2 and SARS-CoV in the Spike protein

10 cleavage: The Spike protein of SARS involves two cleavage sites recognized by

11 TMPRSSs, one at arginine 667 and the other at arginine 797 (right). Compared

12 with SARS-CoV, the Spike protein of SARS-CoV-2 (left) has an insertion

13 sequence $680-$ SPRR-683 at the S1/S2 cleavage site. We speculated that R682,

14 R683 and R685 (red box) could be used as the most suitable substrates for

15 TMPRSSs, which can increase the Spike protein cleavage efficiency of TMPRSSs,

16 promote its activation and enhance SARS-CoV-2 infection. 


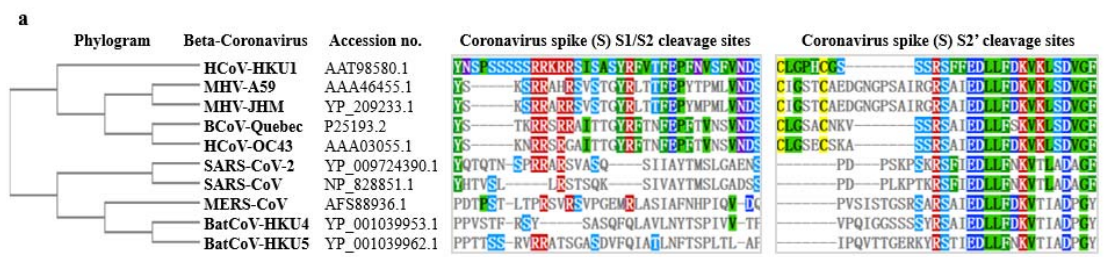

\begin{tabular}{|c|c|c|c|c|}
\hline Beta-Coronavirus & S1/S2 sequence & & S2' sequence & Furin score \\
\hline HCoV-HKU1 & ${ }^{753}$ SSSRRKRRSISASYRF 768 & 0.882 & :97 PHCGSSSRSFFEDLLF 912 & 0.127 \\
\hline MHV-A59 & 714 SRSRRAHRSVSTGYRL 729 & 0.814 & $\approx 66$ GPSAIRGRSAIEDLLF $8 \otimes 1$ & 0.190 \\
\hline MHV-JHM & 762 SKSRRARRSVSTGYRL 777 & 0.887 & 914 GPSAIRGRSAIEDLLF 929 & 0.190 \\
\hline BCoV-Quebec & 761 STKRRSRRSITTGYRF 776 & 0.861 & 906 DCNKVSSRSAIEDLLF 921 & 0.179 \\
\hline HCoV-OC43 & ${ }^{751}$ SRNRRSRGAITTGYRF ${ }^{766}$ & 0.539 & 896 ECSKASSRSAIEDLIF 911 & 0.149 \\
\hline SARS-CoV-2 & 675 QTQTNSPRRARSVASQ 690 & 0.688 & 807 DPSKPSKRSFIEDLLF 823 & 0.328 \\
\hline SARS-CoV & 661 HTVSLIRSTSQ 671 & 0.139 & 790 DPLKPTKRSFIEDLLF 805 & 0.254 \\
\hline MERS-CoV & 744 TLTPRSVRSVPGEMRL 759 & 0.565 & 890 STGSRSARSAIEDLLF 895 & 0.731 \\
\hline BatCoV-HKU4 & 745 VSTFRSYSASQ 755 & 0.163 & 879 GGSSSSYRSAIEDLLF 894 & 0.268 \\
\hline BatCoV-HKU5 & 738 TTSSRVRRATSGASDV 753 & 0.797 & 877 TTGERKYRSTIEDLLF 892 & 0.502 \\
\hline
\end{tabular}

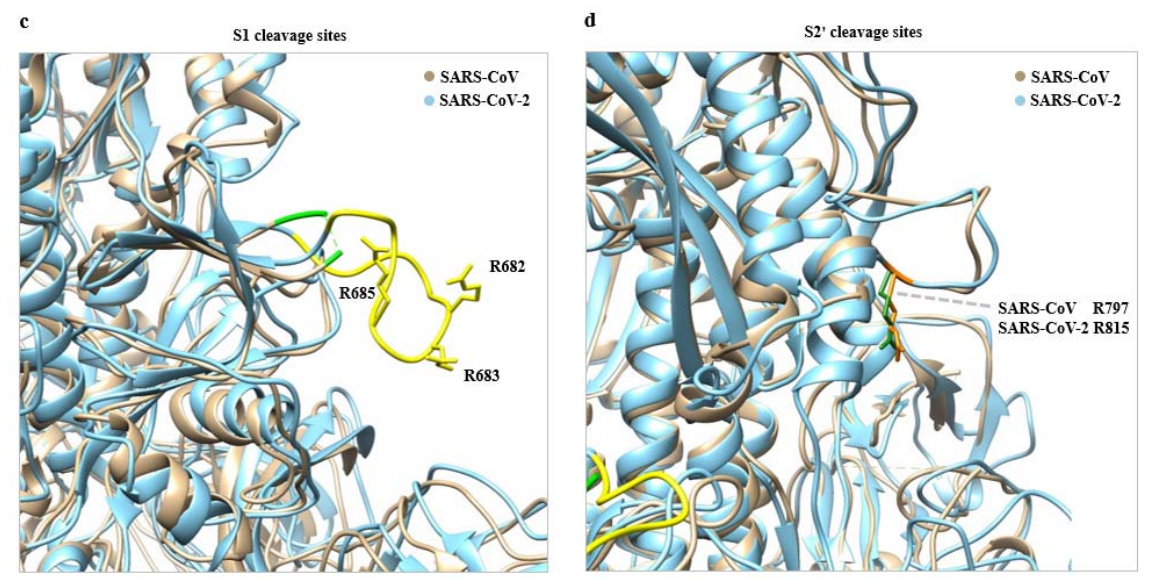

2 Fig. 2 The two potential Spike protein cleavage sites of SARS-CoV and 3 SARS-CoV-2 by TMPRSS2.

4 a. Phylogenetic tree based on the protein sequences of Spike protein in SARS-CoV-2, $5 \quad$ SARS-CoV and other eight beta-coronaviruses are presented, along with the 6 amino acid sequence alignment of two potential cleavage sites by TMPRSS2.

7 b. The putative furin scores of the two potential cleavage sites of the ten coronaviruses.

9 c. Structure comparison of the detailed Spike protein of the SARS-CoV and SARS-CoV-2. The insert 675-690 of SARS-CoV-2 Spike protein (yellow) and the corresponding loci to SARS-CoV Spike protein 661-672 (green). Three important 
1 residues, R682, R683, R685, are specially marked.

2 d. The detail of c. The similarly SARS-CoV R797 with SARS-CoV-2 R815 are

3 marked with forest green and orange, respectively.

4

a

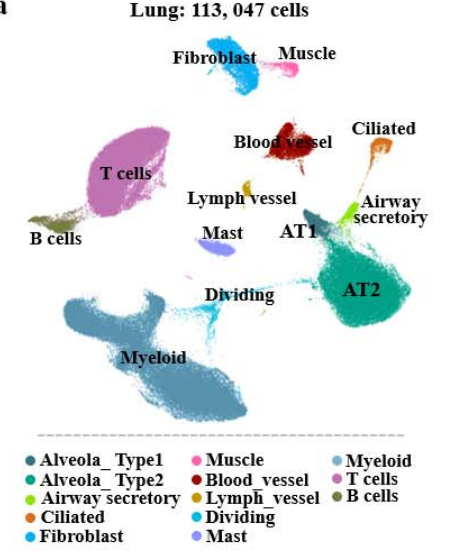

Myeloid cells: $\mathbf{3 5 ,} 148$ cells

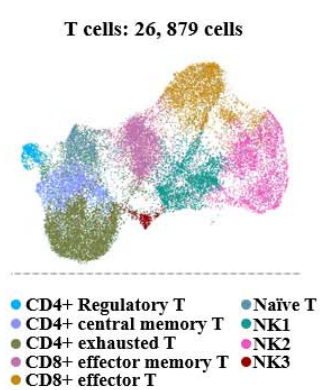

- CD8+ effector memory T $\bullet$ NK3 - CD8+ effector T



b Expression of

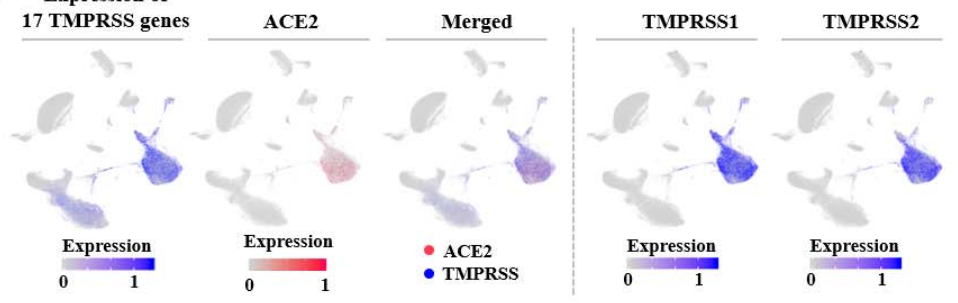

c

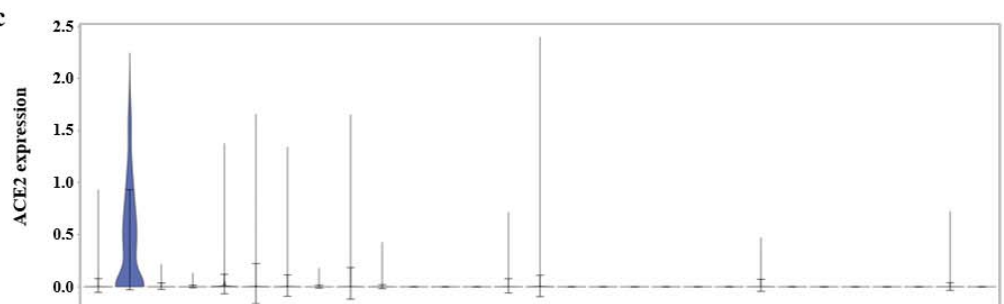

d

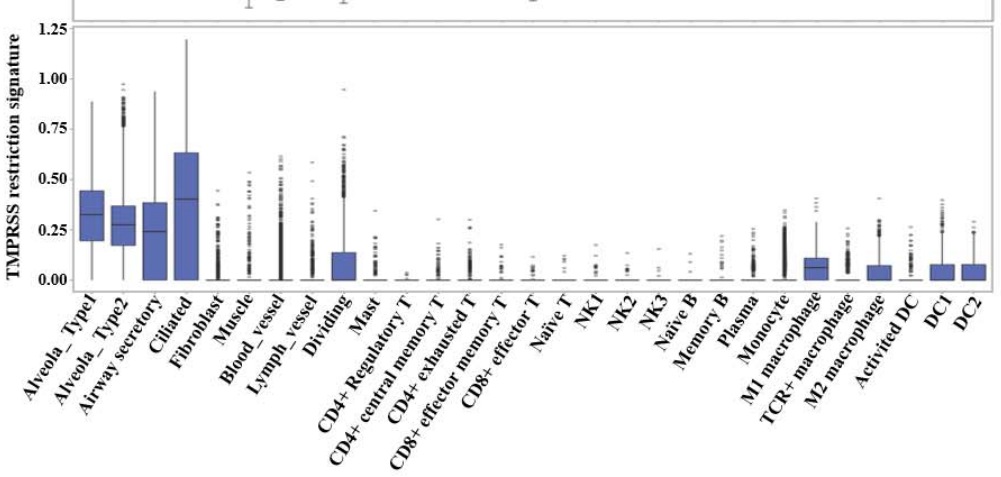

6 Fig. 3 Single-cell analysis of the normal lung tissue. 
1 a. The UMAP plots of the landscape of lung cells. Thirteen clusters are colored, 2 distinctively labeled. T, B and myeloid cell subsets are further divided into finer 3 cell subsets according to the heterogeneity within the cell population.

4 b. The feature plots of the 17 TMPRSS genes, ACE2, TMPRSS1 and TMPRSS2.

5 c. The expression of ACE2 across clusters in the violin plot. The expression is 6 measured as the $\log 2(\mathrm{TP} 10 \mathrm{~K}+1)$ value.

7 d. The mean expression of TMPRSS family genes across clusters in the boxplot. The expression is measured as the mean $\log 2(\mathrm{TP} 10 \mathrm{~K}+1)$ value.
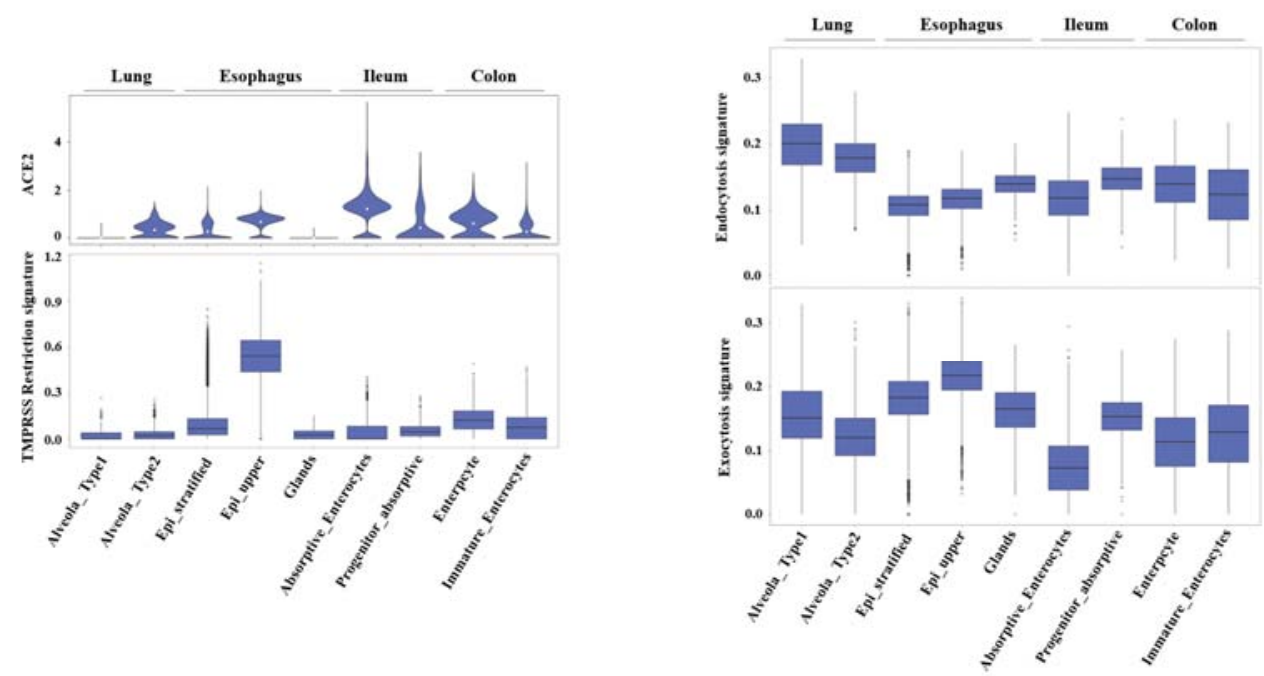

Fig. 4 Expression levels of ACE2, TMPRSS restriction signature and functional gene sets in lung and digestive tracts.

a. The expression levels of ACE2 and TMPRSS restriction signature in 2 lung clusters and 7 digestive tract clusters. The expression is measured as the $\log 2$ $(\mathrm{TP} 10 \mathrm{~K}+1)$ value.

b. The expression levels of endocytosis and exocytosis-associated genes in 2 lung clusters and 7 digestive tract clusters. The expression is measured as the $\log 2$ $(\mathrm{TP} 10 \mathrm{~K}+1)$ value. 




2 Extended Data Fig. 1 The overall structure of the Spike protein in SARS-CoV 3 and SARS-CoV-2 homo-trimers

4 a. The structure of the SARS-CoV Spike protein (from PDB: 5X5B). The insert aa675-690 to SARS-CoV Spike protein aa661-672 with the structural missed residues are marked with green.

b. The structure of the SARS-CoV-2 Spike protein (Modelled by SWISS-MODEL). The insert aa675-690 of 2019-nCoV Spike protein that corresponds to the insert region of SARS-V Spike protein is marked with yellow.

c. The structural superimpose of Spike protein in the SARS-CoV (yellow) and SARS-CoV-2 (blue). 

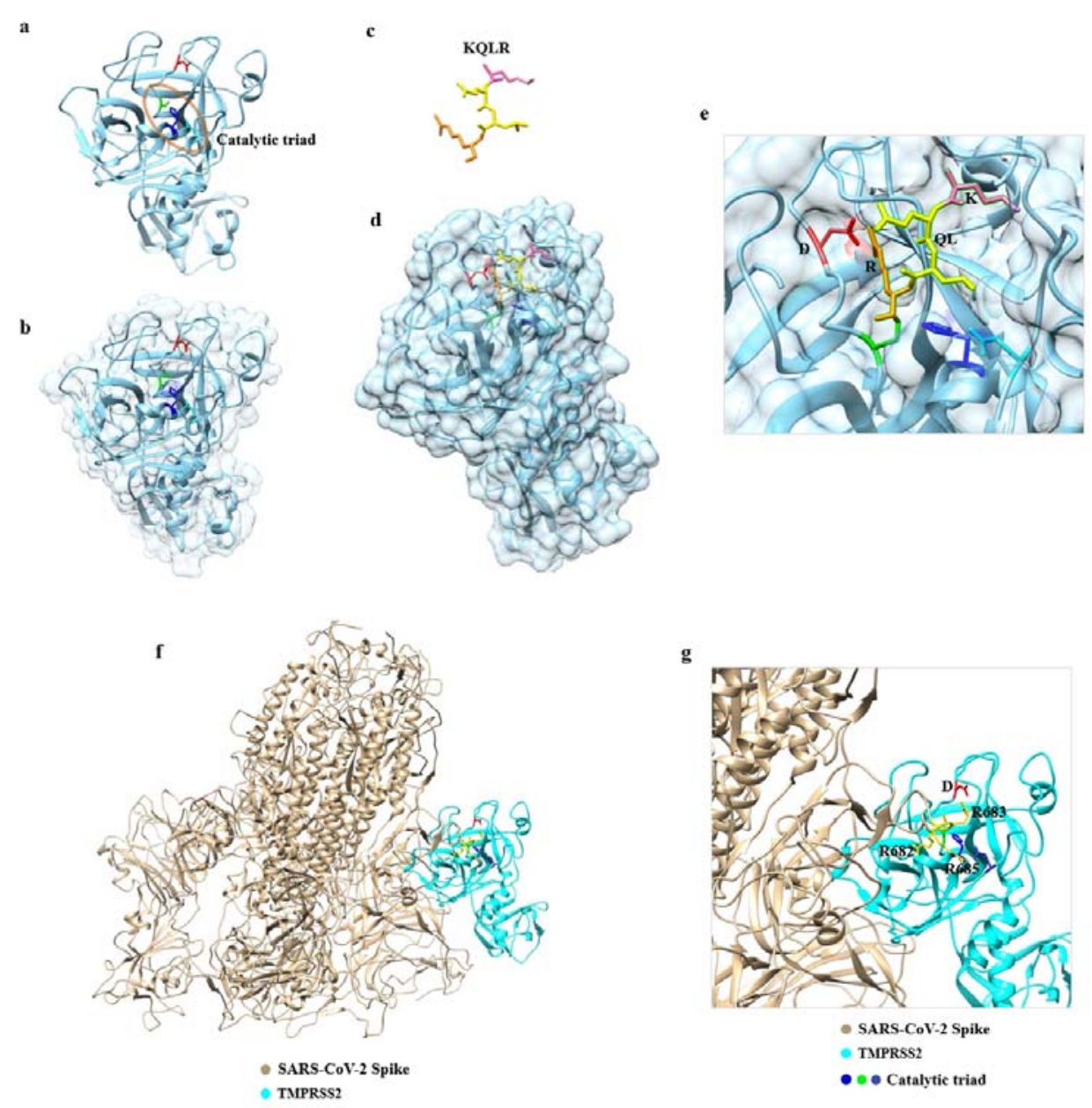

2 Extended Data Fig. 2 The structure and catalytic mechanism of TMPRSS2

3 a-b. The overall structure and surface of TMPRSS2 (Modelled by SWISS-MODEL).

4 The TMPRSS2, catalytic triad comprised of H296, D345 and S441 are marked with

5 cyan, blue, cyan and green, respectively. The substrate binding residue D435 located

6 in the bottom of pocket is marked with red.

7 c. The polypeptide substrate analogue KQLR. The cleavage site Arg is marked with

8 orange. Gln and Leu are marked with yellow. Lys is marked with pink.

9 d. The state of substrate analogue binding in the catalytic pocket. The state of 10 substrate analogue binding in the catalytic pocket.

11 e. The detail of d. Arg of substrate analogue is strongly interacted with D435 
1 f. The predicted state of SARS-CoV-2 Spike protein binding to the catalytic pocket of

2 TMPRSS2.

3 g. The detail of f. SARS-CoV-2 Spike protein and D345 of TMPRSS2 are marked

4 with wheat and medium blue, respectively.

5

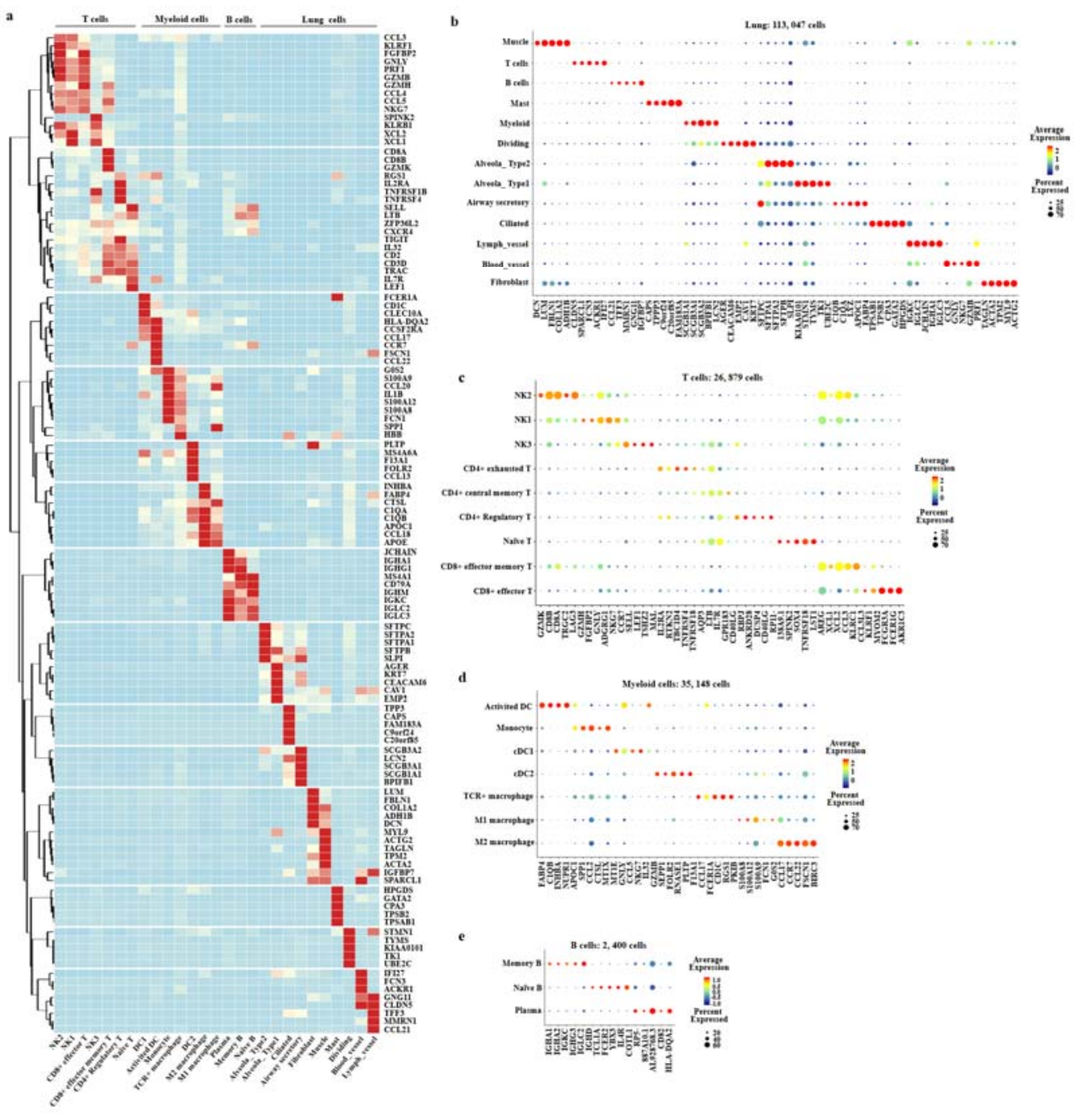

7 Extended Data Fig. 3 Subset-specific markers.

8 a. The heatmap of marker genes (rows) across cell subsets (columns). The bubble

9 diagram of marker genes in thirteen clusters (b) and the sub-clusters of T cells (c),

10 B cells (d) and Myeloid cells (e). 

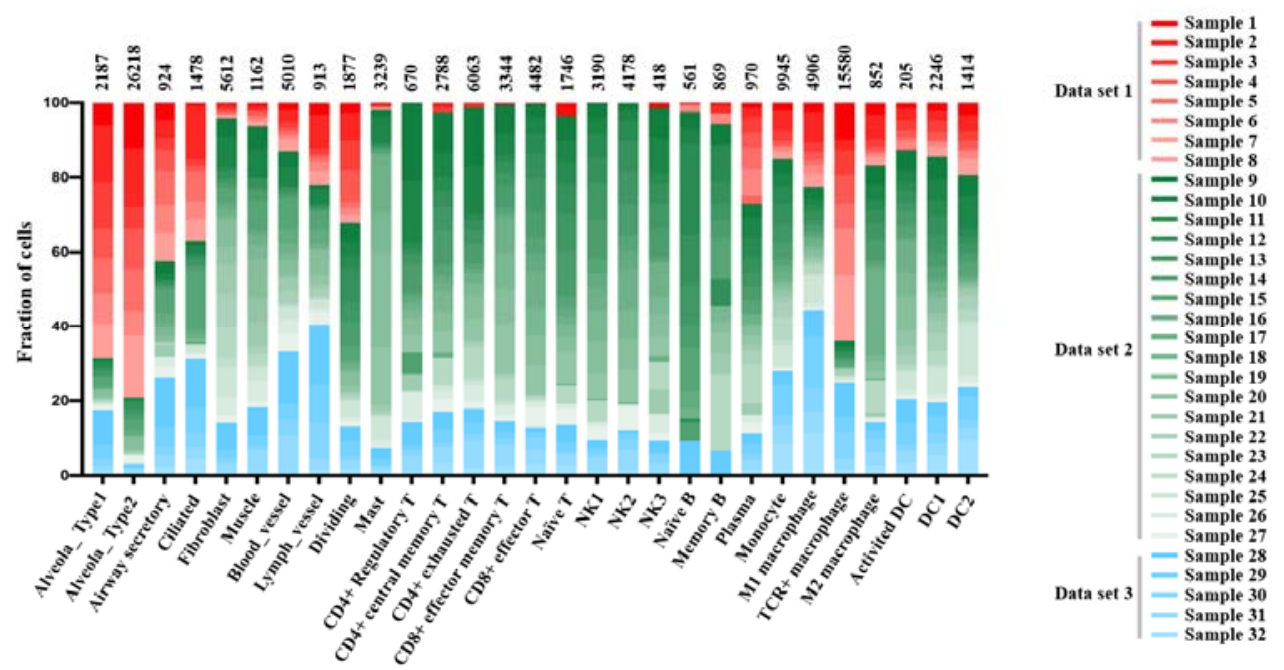

2 Extended Data Fig. 4 All cell subset distributions across samples.

3 The fractions of cells (y axis) in each cell subset (bars) that are derived from each

4 sample in 3 databases (red, green and blue). The numbers of cells in each cluster are

5 labeled above. 

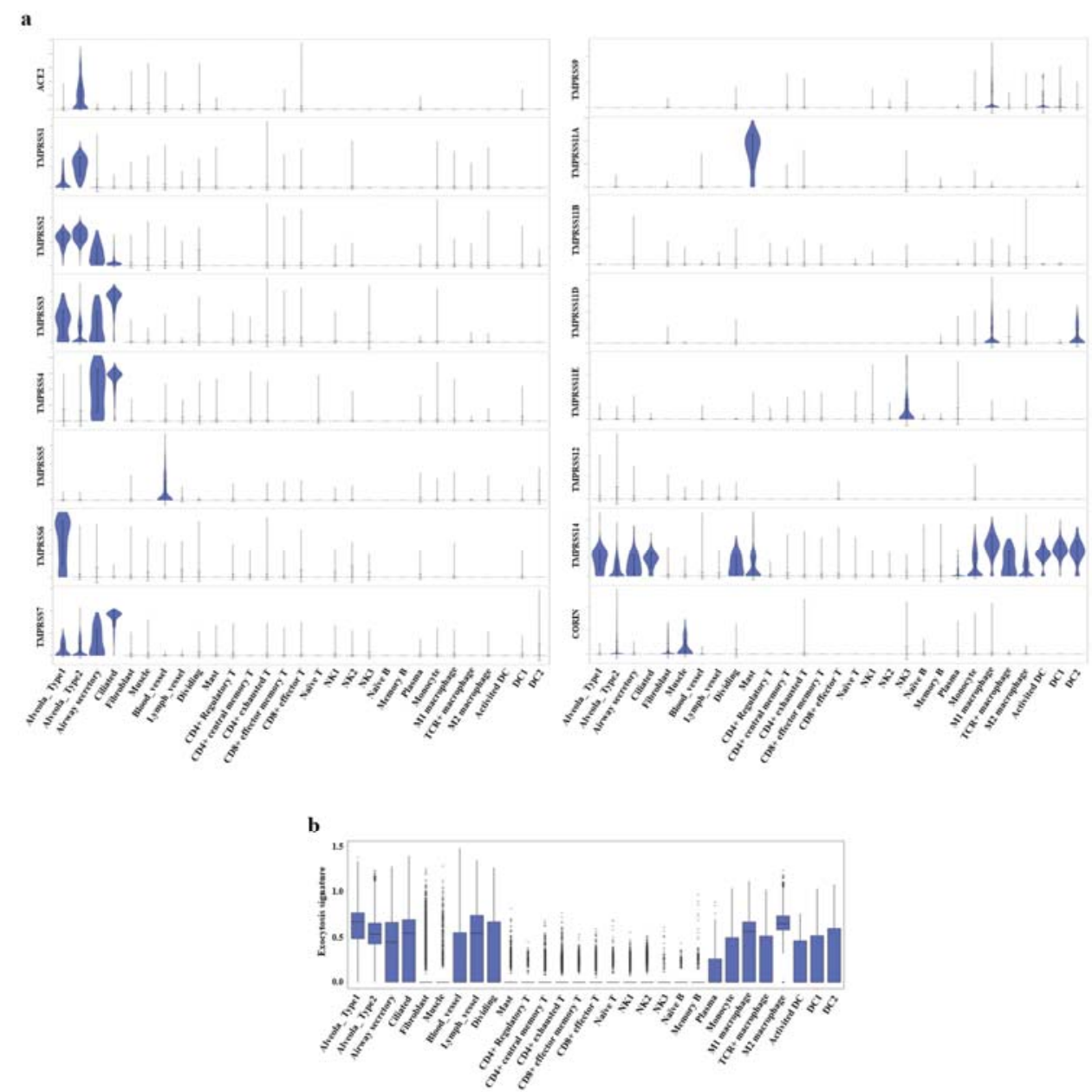

2 Extended Data Fig. 5 The expression levels of ACE2, TMPRSS genes and 3 exocytosis-associated genes in lung subsets.

4 a. The Violin plots of ACE2 and TMPRSS family genes across clusters. The expression is measured as the $\log 2(\mathrm{TP} 10 \mathrm{~K}+1)$ value.

6 b. The boxplot of exocytosis-associated across clusters. The expression is measured $7 \quad$ as the mean $\log 2(\mathrm{TP} 10 \mathrm{~K}+1)$ value. 


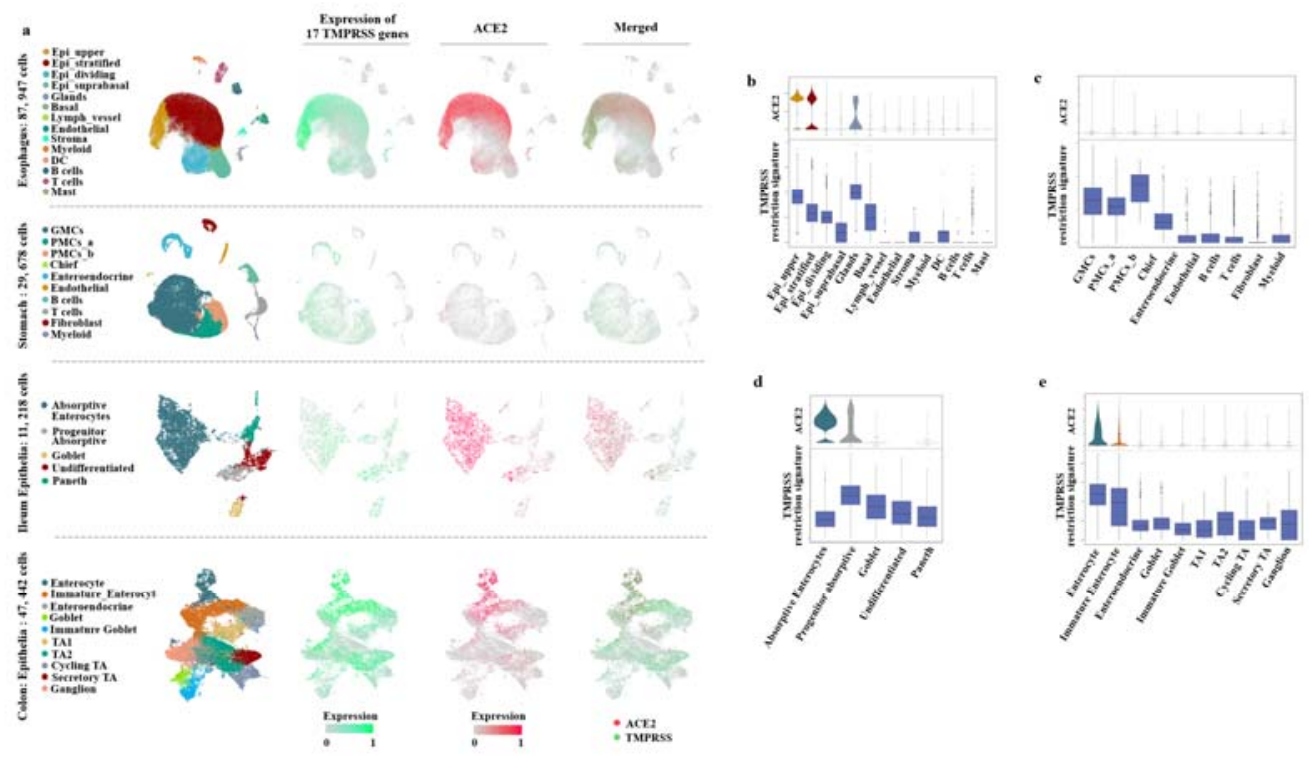

2 Extended Data Fig. 6 The single-cell analysis of esophageal cells, gastric mucosal

3 cells, ileal epithelial cells and colonic epithelial cells.

4 a. The UMAP plots of esophageal cells, gastric mucosal cells, ileal epithelial cells 5 and colonic epithelial cells. The Feature plots show the expression of ACE2 (red) 6 and TMPSS family genes (green). The plots were merged to reveal the 7 co-expression of these genes (brown).

8 c. The expression levels ACE2 and TMPRSS restriction signature across clusters in 9 esophagus (b), stomach(c), ileum(d) and colon(d). The expression is measured as 10 the mean $\log 2(\mathrm{TP} 10 \mathrm{~K}+1)$ value. 



' 1

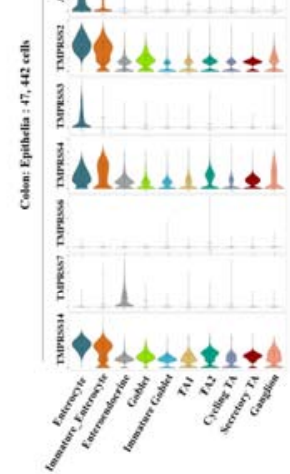

2 Extended Data Fig. 7 The expression levels of ACE2 and TMPRSS family genes

3 in lung and digestive tracts.

4 The violin plots of ACE2 and TMPRSS family genes across clusters in esophagus (a),

5 stomach (b), ileum (c) and colon (d). The expression is measured as the mean $\log 2$

$6(\mathrm{TP} 10 \mathrm{~K}+1)$ value.

7

8 

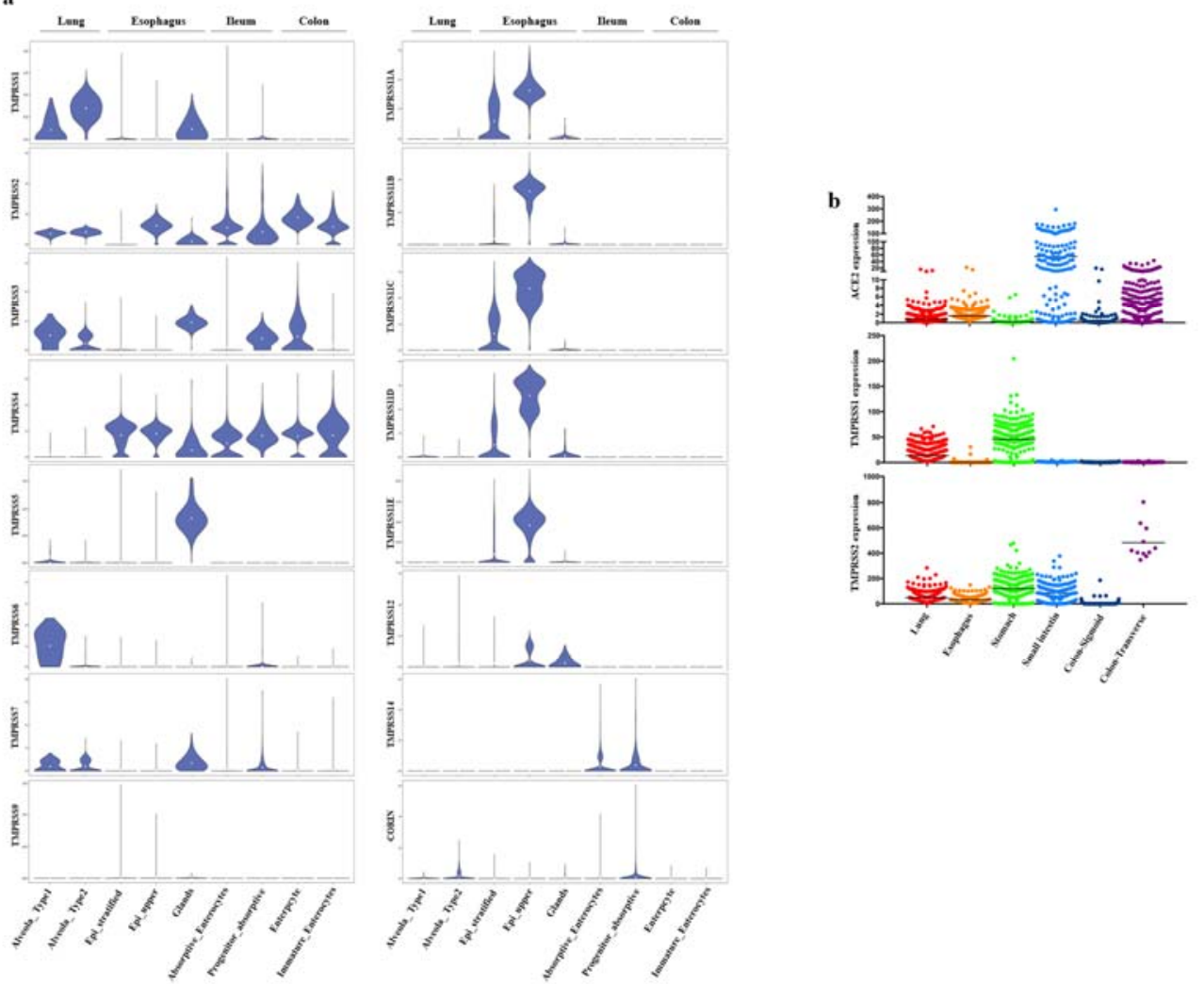

2 Extended Data Fig. 8 The expression levels of ASE2 and TMPRSS family genes

\section{3 in lung and digestive tracts}

4 a. The violin plots of TMPRSS family genes in lung and digestive tracts. The expression is measured as the mean $\log 2(\mathrm{TP} 10 \mathrm{~K}+1)$ value.

6 b. The expression levels of ACE2, TMPRSS1 and TMPRSS2 verified by RNA-seq

7 data from the GTEx database. 